Perez-Roux, T. Transition professionnelle. In A. Jorro (sdr.) Dictionnaire des concepts de la professionnalisation. Bruxelles : de Boeck, pp. 321-324.

\title{
Transition professionnelle
}

Thérèse Perez-Roux, LIRDEF EA3749, Université Paul-Valéry, Montpellier 3

\section{PRESENTATION DU CONCEPT}

Dans son sens général, la transition correspond au passage d'un état à un autre, d'une situation à une autre. Elle suppose à la fois inscription dans le temps, changement d'espace et transformations. Les individus confrontés à un processus de transition sont amenés à réinterroger leur rapport à l'environnement social, culturel, professionnel, relationnel (Boutinet, 2009), ce qui peut générer un certain nombre de tensions.

Tout au long du cycle de vie, les périodes de transition sont nombreuses et diversifiées. Plus spécifiquement, dans la sphère professionnelle, les situations de transition peuvent renvoyer à des moments différents : passage de l'école ou de la formation à l'emploi, phases d'insertion / socialisation dans de nouveaux contextes de travail, périodes de réorientation professionnelle désirées ou subies (perte d'emploi, transformation des prescriptions), etc.

Les phénomènes de transition professionnelle sont aussi en rapport avec les évolutions de la société. Les individus confrontés à des mutations socio-économiques et culturelles doivent trouver des formes de réponses acceptables pour eux-mêmes et en adéquation avec les attentes du monde professionnel : «dans les contextes où de fortes incertitudes traversent les conditions d'accomplissement de soi, les transitions professionnelles et personnelles constituent dès lors des moments particulièrement cruciaux de la socialisation des activités individuelles et des restructurations institutionnelles » (Megemont et Baubion-Broye, 2001, p. 16).

Face à l'hétérogénéité des situations de transitions, il convient de les caractériser à partir de cinq indicateurs proposés par Dupuy et Le Blanc (2001) :

- la transition évoque un processus de développement, vecteur essentiel de changement, qui suppose de prendre en compte les temporalités. L'adaptation des individus au nouvel environnem !ent implique la transformation de certaines valeurs, l'appropriation de nouveaux rôles et à travers eux une modification de l'image de soi, l'acquisition de nouvelles compétences, voire un changement de style de vie ;

- la transition entraîne souvent un changement mais ne peut en aucun cas s'y réduire : entre moments de déstabilisation et de relative stabilité, elle articule continuité et rupture, changement et résistance au changement, combinant de façon dynamique « logiques de scission, d'élimination et de conservation » (p. 63) ;

- la transition questionne différemment les logiques de la reproduction sociale et conduit à des formes de distanciation : de nouvelles orientations individuelles et collectives peuvent être envisagées à partir de situations vécues comme déséquilibrées ou insatisfaisantes ;

- en amenant de nouvelles nécessités et contraintes, la transition pousse les individus et les groupes à développer des stratégies de régulation et d'adaptation ;

- par la combinaison de cadres de référence, de modes de pensée et d'action anciens et nouveaux, la transition génère des formes de remaniement identitaire. 
Perez-Roux, T. Transition professionnelle. In A. Jorro (sdr.) Dictionnaire des concepts de la professionnalisation. Bruxelles : de Boeck, pp. 321-324.

\section{DEBATS AUTOUR DU CONCEPT}

Plusieurs orientations disciplinaires permettent d'appréhender les transitions professionnelles.

Tout d'abord, la perspective anthropologique proposée par Godelier en 1987 ouvre sur une approche socio-économique du phénomène. Cet auteur observe les transitions professionnelles à partir des évolutions sociétales : les rapports socio-économiques antérieurs rencontrant des difficultés internes ou externes pour perdurer, de nouvelles logiques de fonctionnement se généralisent plus ou moins rapidement et conduisent les individus à s'adapter.

Dans une perspective socio-structurelle, centrée sur les politiques et les stratégies des acteurs, la transition professionnelle est étudiée à travers la manière dont les individus trouvent ou construisent de nouvelles logiques d'action et de mobilisation (Zouani-Denoux, 2005).

L'approche psycho-sociale des phénomènes de transition professionnelle est sans aucun doute la plus développée. Elle se centre sur les changements auxquels sont confrontés les individus, sur les ajustements qu'ils opèrent pour donner du sens à ces périodes. Elle s'intéresse par ailleurs aux stratégies déployées pour «faire face à » ou «faire avec »ces modifications. Cette manière d'appréhender les transitions à l'échelle des sujets s'inscrit dans deux perspectives « concurrentes » :

- une perspective développementale : représentée par le paradigme du cours de vie (Elder, 1994), elle décrit les transitions comme des périodes de passage fondamentales et normales dans le cycle de vie. Ces périodes relativement condensées dans le temps, enchâssées dans des trajectoires plus longues, sont considérées comme des phases de co-construction entre individu et environnement passant par des moments d'épreuve ou de crise. Cette approche est remise en cause par des auteurs qui notent des stabilités dans les attitudes des individus en phase transitaire, stabilités préservées dans des interactions avec l'environnement. S'intéressant aux processus de personnalisation, ils revendiquent une approche plus focalisée sur les micro-comportements en s'attachant au sens que donnent les individus aux situations ;

- une perspective interactionniste dans laquelle l'individu est considéré comme participant de façon active au changement social : il utilise donc la transition pour faire des choix dans un environnement qui se transforme. Saisissant contraintes et opportunités du contexte, il décide, s'engage, se met en projet. La transition est alors envisagée comme une chance de développement de la carrière ou comme moyen de renforcer/retrouver ses valeurs, comme une "phase de reconstruction active des valeurs et des normes fondant la reconnaissance et la valorisation de soi et autrui » (Dupuy et Le Blanc, 2001, p. 68).

Au plan épistémologique, on repère deux positions : d'un côté, une approche des transitions envisageant le changement comme extérieur au sujet ou inscrit dans des étapes de développement et d'intégration supposées connues (logique développementale) ; de l'autre, une prise en compte de la responsabilité du sujet dans ce processus, amenant à considérer les transitions «comme des espaces-temps de co-construction du changement individuel et social » (Dupuy et Le Blanc, 2001, p. 74) dans lesquels les trajectoires socio-professionnelles sont liées à la fois aux contextes socio-économiques et culturels mais aussi aux stratégies singulières intégrant représentations, valeurs, rôles et modes d'implication professionnelle. 
Perez-Roux, T. Transition professionnelle. In A. Jorro (sdr.) Dictionnaire des concepts de la professionnalisation. Bruxelles : de Boeck, pp. 321-324.

Au plan méthodologique, l'étude des transitions suppose de s'inscrire dans des temporalités longues pour saisir les processus à l'œuvre du côté des sujets. Si les approches qualitatives et compréhensives semblent incontournables, elles ne peuvent faire l'économie des rapports des individus aux collectifs et à l'organisation. Enfin elles supposent de tenir compte des systèmes politiques, sociaux et culturels dans lesquels s'inscrit la transition.

Enfin, parler de transition professionnelle amène à envisager ses liens : d'une part avec les processus de remaniement identitaire (Balleux et Perez-Roux, 2011) que génère tout changement; d'autre part avec les modalités d'accompagnement mises en œuvre en formation. Cette perspective relève d'enjeux professionnels importants. Là encore, la question des temporalités semble essentielle pour réussir à la fois, dans ce temps de la transition, appropriation de savoirs nouveaux, socialisation professionnelle et activité de personnalisation permettant de prendre en compte les ancrages personnels mis en tension, les leviers et les obstacles à l'engagement tant personnel que professionnel (Zitoun et PerretClermont, 2001).

\section{BIBLIOGRAPHIE}

Balleux, A. et Perez-Roux, T. (2011). Transitions professionnelles et recompositions identitaires. Recherches en Education, 11. 5-14 http://www.recherches-eneducation.net/IMG/pdf/REE-no11.pdf.

Boutinet, J. P. (2009). Transition adulte, L'ABC de la VAE. Paris : Éditions Éres.

Dupuy, R. et Le Blanc, A. (2001). Enjeux axiologiques et activités de personnalisation dans les transitions professionnelles. Connexions, 2 (76), 61-79.

Elder, G.H. Jr. (1994). Time, human agency, and social change : perspectives on the life course. Social Psychology Quaterly, 57 (1), 4-15.

Godelier, M. (1987). Introduction : L'analyse des processus de transition. Revue internationale des sciences sociales, 114, 501-512.

Mègemont, J-L. et Baubion-Broye A. (2001). Dynamiques identitaires et représentations de soi dans une phase de transition professionnelle et personnelle. Connexions, 2 (76), 15-28.

Zittoun, T. et Perret-Clermont A.N. et (2001). Contributions à une psychologie de la transition. Communication présentée dans le cadre du Congrès international de la Société suisse pour la recherche en éducation (SSRE) et de la Société suisse pour la formation des enseignantes et des enseignants (SSFE), Aarau : 5 octobre 2001.

Zouani-Denoux, S. (2005). La transition professionnelle par alternance. Projets et stratégies du formé. Carrefours de l'éducation, 20, 123-138 